\title{
Cryptanalysis of Bluetooth Keystream Generator Two-Level E0
}

\author{
Yi Lu* and Serge Vaudenay \\ EPFL \\ http://lasecwww.epfl.ch
}

\begin{abstract}
In this paper, we carefully study both distinguishing and key-recovery attacks against Bluetooth two-level E0 given many short frames. Based on a flaw in the resynchronization of Bluetooth E0, we are able to fully exploit the largest bias of the finite state machine inside E0 for our attacks. Our key-recovery attack works with $2^{40}$ simple operations given the first 24 bits of $2^{35}$ frames. Compared with all existing attacks against two-level E0, this is the best one so far.
\end{abstract}

\section{Background}

The short-range wireless technology Bluetooth uses the keystream generator E0 to produce the keystream for encryption. After the earlier results [10, 9, 6] of correlation (also called bias) properties inside the Finite State Machine (FSM) towards the one-level E0, most recently, [12] systematically studied the biases and proved two previously known large biases to be the only largest up to 26 consecutive bits of the FSM output sequences. Attacks against E0 mostly focus on one-level E0 only and the best attacks [12, 1, 5, work on one impractically long frame of keystream without exception. Nevertheless, a few attacks [15, 11, 17-9] apply to two-level E0; compared with feasible attack complexities on one-level E0, attack complexities on two-level E0 are extremely high and make the practical Bluetooth E0 unbroken.

The main contribution of this paper is that first based on one of the two largest biases inside the FSM within one-level E0, we identify the bias at twolevel E0 due to a resynchronization flaw in Bluetooth E0. Unlike the traditional approach to find the bias, the characterized bias does not involve the precomputation of the multiple polynomial with low weight. Second, to utilize the identified bias, we develop a novel attack to directly recover the original encryption key for two-level E0 without reconstructing the initial state of E0 at the second level. Our key-recovery attack works with $2^{40}$ simple operations given the first 24 bits of $2^{35}$ frames. Compared with all existing attacks [15, 11, 7,-9] against two-level E0, this is the best so far.

\footnotetext{
* supported in part by the National Competence Center in Research on Mobile Information and Communication Systems (NCCR-MICS), a center of the Swiss National Science Foundation under the grant number 5005-67322.
} 


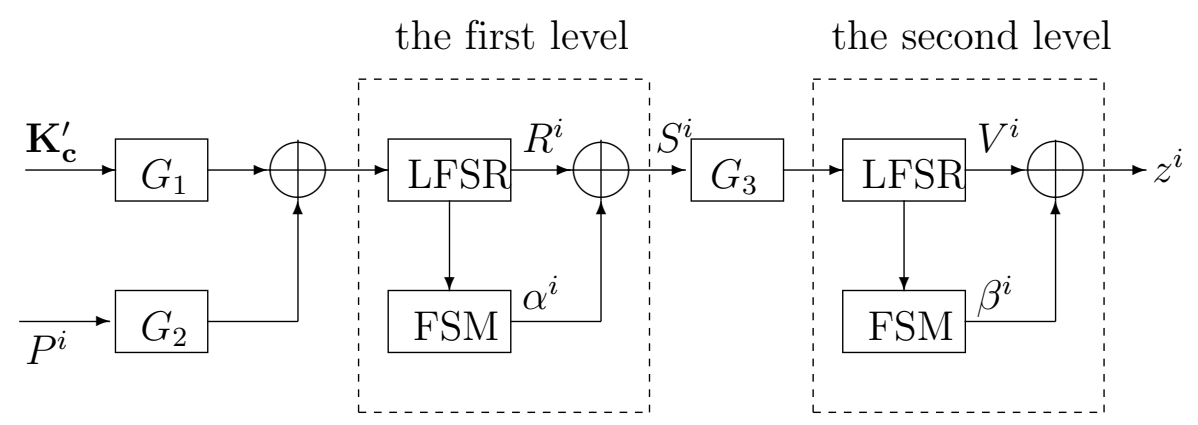

Fig. 1. Diagram of two-level E0 keystream generation

The rest of the paper is structured as follows. In Section 2 we review description of two-level E0. In Section 3 we study the attack against one-level E0. Then, we investigate the E0 resynchronization flaw, which allows to develop the basic attack of previous section into the distinguishing and key-recovery attacks against two-level E0 in Section 4. we further extend our key-recovery attack in Section 5 . Finally, we conclude in Section 6 .

\section{$2 \quad$ Preliminaries}

\subsection{The Core of Bluetooth E0}

To briefly outline, the core of E0 (both dashed boxes in Fig. 1) can be viewed as a nonlinear filtering generator. The filtering generator consists of four LFSRs $\left(R_{1}, \ldots, R_{4}\right)$ which are equivalent to a single $L$-bit LFSR with connection polynomia $11 p(x)$ and a 4-bit FSM, where $L=128$. The keystream bit of the generator is obtained by xoring the output bit of the regularly-clocked LFSR with that of the FSM, which takes the current state of the LFSR as input and emits one bit (denoted by $c_{t}^{0}$ in Bluetooth specification) out of its 4-bit memory.

\subsection{Review on Two-Level Bluetooth E0}

Let $\mathbf{K}_{\mathbf{c}}$ be the $L$-bit secret key computed by the key generation algorithm E3 [3. p783]. According to [3], the effective key $\mathcal{K}$ of length $8 \ell(1 \leq \ell \leq 16)$ is computed by

$$
\mathcal{K}(x)=\mathbf{K}_{\mathbf{c}}(x) \bmod g_{1}^{(\ell)}(x),
$$

where the polynomial $g_{1}^{(\ell)}(x)$ is specified in [3, p770] and has degree $8 \ell$. Bluetooth two-level E0 (depicted in Fig. 1) uses two $L$-bit inputs: one is the known nonce 2

${ }^{1}$ Note that the connection polynomial of the equivalent single LFSR equals the product of those of the four LFSRs.

${ }^{2}$ By convention, hereafter we always use the superscript $i$ to indicate the context of the $i$-th frame. 
$P^{i}$, the other is the linearly expanded $L$-bit key $\mathbf{K}_{\mathbf{c}}^{\prime}$ from $\mathcal{K}$ by $\mathbf{K}_{\mathbf{c}}^{\prime}(x)=g_{2}^{(\ell)}(x)$. $\mathcal{K}(x)$, where the polynomial $g_{2}^{(\ell)}(x)$ is also specified in [3, p770] and has degree no larger than $L-8 \ell$; or equivalently, we can rewrite it as

$$
\mathbf{K}_{\mathbf{c}}^{\prime}=E(\mathcal{K}),
$$

where $E$ is a linear mapping. After initialization of the equivalent LFSR for the first level E0, we can express its initial state $R_{[-199, \ldots, L-200]}^{i}=\left(R_{-199}^{i}, \ldots\right.$, $\left.R_{L-200}^{i}\right)$ as

$$
R_{[-199, \ldots, L-200]}^{i}=G_{1}\left(\mathbf{K}_{\mathbf{c}}^{\prime}\right) \oplus G_{2}\left(P^{i}\right),
$$

for $i=1, \ldots, m$, where $G_{1}$ and $G_{2}$ are affine transformations over $G F(2)^{L}$. Next comes the so-called two-level E0:

- During the first level, with the FSM initial state preset to zero, E0 runs $L$ clocks producing 200-bit output $S_{t}^{i}=R_{t}^{i} \oplus \alpha_{t}^{i}$ and updating $R_{[t, \ldots, t+L-1]}^{i}$ by $R_{[t+1, \ldots, t+L]}^{i}=M\left(R_{[t, \ldots, t+L-1]}^{i}\right)$ for $t=-199, \ldots, 0$, where $M$ is the linear mapping over $G F(2)^{L}$ that corresponds to the companion matrix associated with $p(x)$. Note that first, $\alpha_{t}^{i}$ is the output bit of the FSM fed with $R_{[t, \ldots, t+L-1]}^{i}$; second, the last $L$-bit output at the first level E0 is $S_{[1-L, \ldots, 0]}^{i}$; last, $R_{[1-L, \ldots, 0]}^{i}=\left(M^{72} \circ G_{1}\right)\left(\mathbf{K}_{\mathbf{c}}^{\prime}\right) \oplus\left(M^{72} \circ G_{2}\right)\left(P^{i}\right)$.

- At the beginning of the second level, the equivalent single LFSR is initialized by $V_{[1, \ldots, L]}^{i}=G_{3}\left(S_{[1-L, \ldots, 0]}^{i}\right)$, where $G_{3}: G F(2)^{128} \rightarrow G F(2)^{128}$ is another affine transformation (see [3, p772]); the FSM initial state at the second level remains the same as the one in the end of the first level. Note that the present time is $t=1$.

- During the second level, for $t=1, \ldots, 2745$, E0 produces the keystream $z_{t}^{i}=V_{t}^{i} \oplus \beta_{t}^{i}$ for encryption of the $i$-th frame and updates $V_{[t, \ldots, t+L-1]}^{i}$ by $V_{[t+1, \ldots, t+L]}^{i}$.

\subsection{An Important Note on $\boldsymbol{G}_{3}$}

We observe that $G_{3}$ is implemented in such a simple way that the last $L$-bit output sequence of the first level $\mathrm{E} 0$ is byte-wise reloaded into the four component LFSRs in parallel at the second level E0 with only a few exceptions, which turns out to be a flaw as introduced later in Section 4 . For completeness, Table 1 lists in time order the first 24 output bits of $R_{1}, \ldots, R_{4}$ individually at the beginning of E0 level two, in terms of the $L$-bit input $v_{0}, \ldots, v_{L-1}$.

\subsection{The Bias Inside the FSM}

Our starting point would be the bias inside the FSM, which was discovered by [9, 6] and further proved in [12] to be the the largest bias up to 26-bit output

${ }^{3}$ Throughout the rest of the paper, we use the unified notation $\Omega_{[a, \ldots, b]}^{i}$ with the formatted subscript to denote the vector $\left(\Omega_{a}^{i}, \ldots, \Omega_{b}^{i}\right)$.

${ }^{4}$ It is believed to help increase the rate of keystream generation. 
Table 1. The first 24 output bits of LFSRs at E0 level two

\begin{tabular}{|c|c|c|}
\hline LFSR & \multicolumn{2}{|c|}{ output bits } \\
\hline$R_{1}$ & $v_{71} \cdots v_{64}, v_{39} \cdots$ & $v_{32}, v_{7} \cdots v_{0}$ \\
\hline$R_{2}$ & $\begin{array}{lllll}v_{79} & \cdots & v_{72}, & v_{47} & \cdots \\
\end{array}$ & $v_{40}, v_{15} \cdots v_{8}$ \\
\hline$R_{3}$ & $v_{111} \cdots v_{104}, v_{87}$ & $v_{80}, v_{55} \cdots v_{48}$ \\
\hline$R_{4}$ & $v_{119} \cdots v_{112}, v_{95}$ & $v_{88}, v_{63} \cdots v_{56}$ \\
\hline
\end{tabular}

sequence of the FSM involving the smallest number of consecutive bits. Let $\lambda=\frac{25}{256}$, we have

$$
\operatorname{Pr}\left(c_{t}^{0} \oplus c_{t+1}^{0} \oplus c_{t+2}^{0} \oplus c_{t+3}^{0} \oplus c_{t+4}^{0}=1\right)=\frac{1}{2}+\frac{\lambda}{2},
$$

for any integer $t$, assuming that the $L+4=132$-bit initial state of E0 is random and uniformly distributed. Hereafter, we analyze as exactly described in Bluetooth specification [3]. For convenience, we denote $c_{t}^{0}$ used for the first and second level keystream generation by $\alpha_{t}^{i}, \beta_{t}^{i}$ respectively. Therefore $\left\{\alpha_{t}^{i}\right\},\left\{\beta_{t}^{i}\right\}$ being separated sequences of $\left\{c_{t}^{0}\right\}$ both satisfy the same statistical property:

$$
\begin{aligned}
& \operatorname{Pr}\left(\alpha_{t}^{i} \oplus \alpha_{t+1}^{i} \oplus \alpha_{t+2}^{i} \oplus \alpha_{t+3}^{i} \oplus \alpha_{t+4}^{i}=1\right)=\frac{1}{2}+\frac{\lambda}{2}, \\
& \operatorname{Pr}\left(\beta_{t}^{i} \oplus \beta_{t+1}^{i} \oplus \beta_{t+2}^{i} \oplus \beta_{t+3}^{i} \oplus \beta_{t+4}^{i}=1\right)=\frac{1}{2}+\frac{\lambda}{2},
\end{aligned}
$$

for any $t$ and any $i$.

\section{Security Analysis on E0 Level One}

The goal of the attacker in this section is to recover the effective $8 \ell$-bit encryption key $\mathcal{K}$ with knowledge of $m L$-bit output sequences $S_{[1-L, \ldots, 0]}^{i}$ of the first level E0 for $i=1, \ldots, m$ and the corresponding $m$ nonces $P^{1}, \ldots, P^{m}$.

\subsection{Finding the Closest Sequences with Fixed Differences}

We begin with a very simple problem: given $2 m L$-bit sequences $s^{1}, \ldots, s^{m}$ and $\delta^{1}, \ldots, \delta^{m}$, where $\delta^{1}=\mathbf{0}$ and $\delta^{i} \neq \delta^{j}$ for all $i \neq j$, find the $L$-bit sequence $r^{1}$ that maximizes $N\left(r^{1}\right)=\sum_{i=1}^{m} \sum_{t=1}^{L}\left(s_{t}^{i} \oplus r_{t}^{i}\right)$ where $r_{t}^{i}=r_{t}^{1} \oplus \delta_{t}^{i}$ for $i=1, \ldots, m$ and $t=1, \ldots, L$.

Similar to the well-known approach (see 9, p251]), the solution based on the idea of minority vote goes fairly easy. We have

$$
N\left(r^{1}\right)=\sum_{t=1}^{L} \sum_{i=1}^{m}\left(s_{t}^{i} \oplus r_{t}^{1} \oplus \delta_{t}^{i}\right) .
$$

Thus, in order to maximize $N\left(r^{1}\right)$, we must have

$$
r_{t}^{1}=\operatorname{minority}\left\{s_{t}^{i} \oplus \delta_{t}^{i}: i=1, \ldots, m\right\}
$$


for all $t=1, \ldots, L$. Note that in case of a tie for $r_{t}^{1}$, we have two answers for this $t$-th bit regardless of all the other bits. We finally obtain all the answers that achieve the same maximal $N\left(r^{1}\right)$. The time and memory complexities of the above algorithm both equal the data complexity $O(m L)$.

\subsection{Attack Against E0 Level One}

Let $\Delta_{[1-L, \ldots, 0]}^{i}=R_{[1-L, \ldots, 0]}^{1} \oplus R_{[1-L, \ldots, 0]}^{i}$ for $i=1, \ldots, m$. By Eq.(2) we have $\Delta_{[1-L, \ldots, 0]}^{i}=\left(M^{72} \circ G_{2}\right)\left(P^{1} \oplus P^{i}\right)$. We further set

$$
\begin{aligned}
& r_{t}^{i}=\bigoplus_{j=0}^{4} R_{t+j}^{i}, \\
& \delta_{t}^{i}=\bigoplus_{j=0}^{4} \Delta_{t+j}^{i}, \\
& s_{t}^{i}=\bigoplus_{j=0}^{4} S_{t+j}^{i},
\end{aligned}
$$

for $i=1, \ldots, m$ and $t=1-L, \ldots,-4$. Note that $s_{t}^{i} \oplus r_{t}^{i}=\bigoplus_{j=0}^{4} \alpha_{t+j}^{i}$ follows the biased distribution by Eq.(3). As long as $\sum_{i=1}^{m} \sum_{t=1-L}^{-4}\left(s_{t}^{i} \oplus r_{t}^{i}\right)$ is the maximal and $\Delta_{t}^{i}, S_{t}^{i}$ are known, we can apply the preceding algorithm to recover $(L-$ 4) bits of $r^{1}$ followed by an exhaustive search on the remaining 4 bits, next solve $R^{1}$, then $\mathbf{K}_{\mathbf{c}}^{\prime}$ by Eq.(2), and finally deduce $\mathcal{K}$ from $\mathbf{K}_{\mathbf{c}}^{\prime}$ by Eq.(1). The time/memory/data complexities all equal $O\left(m L+2^{4} L\right)$, i.e. $O((m+16) L)$. No precomputation is needed.

About the minimal $m$ to guarantee the valid precondition $\sum_{i=1}^{m} \sum_{t=1-L}^{-4}\left(s_{t}^{i} \oplus\right.$ $\left.r_{t}^{i}\right)$ is the maximal, we use the result in [12, Eq.(10)] based on [2] that says regardless of the value of $L$ and $\ell$, we need the minimum

$$
m \approx \frac{4 \log 2}{\lambda^{2}} \text { (frames). }
$$

Consequently, we require $m=512$ to recover $\mathcal{K}$ from $S^{i}$ and $P^{i}$ for $i=$ $1, \ldots, m$. This results in the time/data/memory complexities all the same as $O\left(2^{16}\right)$. To verify this, we ran experiments on 512 frames of the randomly-chosen 132-bit E0 initial state $2^{25}$ times. It turned out that we had 1.5 errors and 0.4 tie in average, which means we can easily correct all errors by an extra checking step in the end in negligible time. Finally, Table 2 compares our result with the only known 5 four attacks [7, 8, 11, 15] working on frames of $L$-bit consecutive keystreams. Note that existing attacks [7, 8, 11, 15] directly apply to two-level E0

${ }^{5}$ In the similar approached paper [9], $m$ is chosen as 45 for E0 level one without the complexity estimate, because the authors focused on the two-level E0 and traded $m$ with the time complexity of E0 level one, whose time complexity is negligible with that of the E0 level two. 
as well with the level-by-level key-recovery schem\&; in contrast, our attack is completely disabled against two-level E0 with this scheme as the attack is based on a naive assumption that we directly observe the output of E0 level on 7 . In the next section, we introduce a resynchronization flaw in Bluetooth E0 that leads to a shortcut extended attack against the two-level E0.

Table 2. Comparison of our attack with existing attacks against E0 level one given frames of $L$ bits

\begin{tabular}{||cr||c|c|c|c|c||}
\hline \multicolumn{2}{|c|}{ Attack Type } & Precomputation & Time & Frames & Data & Memory \\
\hline \hline Divide \& Conquer & {$[15$} & - & $2^{93}$ & 1 & $2^{7}$ & - \\
\hline BDD & {$[11]$} & - & $2^{77}$ & 1 & $2^{7}$ & - \\
\hline Algebraic Attack & $7]$ & - & $2^{51}$ & 2 & $2^{8}$ & $2^{51}$ \\
\hline Algebraic Attack & $\mathbf{8}$ & - & $2^{23.4}$ & 3 & $2^{8.6}$ & $2^{23.4}$ \\
\hline Our Correlation Attack & - & $2^{16}$ & $2^{9}$ & $2^{16}$ & $2^{16}$ \\
\hline
\end{tabular}

\section{Security Analysis on Two-Level E0}

\subsection{The Resynchronization Flaw in Bluetooth Two-Level E0}

Define

$$
U^{i}=\left(U_{1}^{i}, \ldots, U_{L}^{i}\right)=G_{3}\left(R_{[1-L, \ldots, 0]}^{i}\right) .
$$

Following the description of $G_{3}$ in Subsection 2.3 , we can easily verify that

$$
\begin{aligned}
& V_{t}^{i}=U_{t}^{i} \oplus \alpha_{-56-t}^{i} \oplus \alpha_{-48-t}^{i} \oplus \alpha_{-16-t}^{i} \oplus \alpha_{-8-t}^{i}, \quad \text { for } t=1, \ldots, 8 \text {, } \\
& V_{t}^{i}=U_{t}^{i} \oplus \alpha_{-80-t}^{i} \oplus \alpha_{-72-t}^{i} \oplus \alpha_{-32-t}^{i} \oplus \alpha_{-24-t}^{i} \text {, for } t=9, \ldots, 16 \text {, } \\
& V_{t}^{i}=U_{t}^{i} \oplus \alpha_{-104-t}^{i} \oplus \alpha_{-96-t}^{i} \oplus \alpha_{-56-t}^{i} \oplus \alpha_{-48-t}^{i} \text {, for } t=17, \ldots, 24 \text {. }
\end{aligned}
$$

From the above equations, we summarize the characteristics of $V_{t}^{i}$ by

$$
V_{t}^{i}=U_{t}^{i} \oplus \alpha_{a_{t}}^{i} \oplus \alpha_{a_{t}+8}^{i} \oplus \alpha_{b_{t}}^{i} \oplus \alpha_{b_{t}+8}^{i}
$$

for $t=1, \ldots, 24$, where $a_{t}=-t+$ const $_{\left\lfloor\frac{t-1}{8}\right\rfloor}$ and $b_{t}=-t+$ const $_{\left\lfloor\frac{t-1}{8}\right\rfloor}^{\prime}$. Note that Eq.(7) is our crucial observation about Bluetooth E0 resynchronization flaw which enables a shortcut attack throughout the two levels of E0. Now, we express the output bit $z_{t}^{i}$ of the second level E0 keystream by

$$
z_{t}^{i}=U_{t}^{i} \oplus \alpha_{a_{t}}^{i} \oplus \alpha_{a_{t}+8}^{i} \oplus \alpha_{b_{t}}^{i} \oplus \alpha_{b_{t}+8}^{i} \oplus \beta_{t}^{i}
$$

\footnotetext{
${ }^{6}$ namely, the initial state at the first level is reconstructed after the initial state at the second level is recovered.

7 As a matter of fact, according to [3, p763], Bluetooth takes the correlation properties into account and adopts the two-level scheme of keystream generation in practice on purpose.
} 
for $t=1, \ldots, 24$. Let $u_{t}^{i}=\bigoplus_{j=0}^{4} U_{t+j}^{i}$ and $s_{t}^{i}=\bigoplus_{j=0}^{4} z_{t+j}^{i}$. From Eq.(8), we have that

$$
s_{t}^{i} \oplus u_{t}^{i}=\bigoplus_{j=0}^{4} \alpha_{a_{t}-j}^{i} \oplus \bigoplus_{j=0}^{4} \alpha_{a_{t}-j+8}^{i} \oplus \bigoplus_{j=0}^{4} \alpha_{b_{t}-j}^{i} \oplus \bigoplus_{j=0}^{4} \alpha_{b_{t}-j+8}^{i} \oplus \bigoplus_{j=0}^{4} \beta_{t+j}^{i}
$$

for $t=8 k+1, \ldots, 8 k+4, k=0,1,2$. Therefore, we deduce an important correlation concerning the practical implementation of E0 from Eq.(34.9):

Theorem 1. Assuming independence of $\alpha_{t}^{i}$ 's and $\beta_{t}^{i}$ 's, we have

$$
\operatorname{Pr}\left(s_{t}^{i} \oplus u_{t}^{i}=1\right)=\frac{1}{2}+\frac{\lambda^{5}}{2}
$$

for $t=8 k+1, \ldots, 8 k+4, k=0,1,2$.

\subsection{A Near-Practical Distinguishing Attack Against Two-Level E0}

Using the standard technique of linear cryptanalysis, we expect that $s_{t}^{i} \oplus u_{t}^{i}$ equals one most of the time for $t=8 k+1, \ldots, 8 k+4, k=0,1,2$ with a total of $\lambda^{-10} \approx 2^{34}$ samples. Since the difference

$$
U^{i} \oplus U^{j}=G_{3}\left(R_{[1-L, \ldots, 0]}^{i}\right) \oplus G_{3}\left(R_{[1-L, \ldots, 0]}^{j}\right)=\left(G_{3} \circ M^{72} \circ G_{2}\right)\left(P^{i} \oplus P^{j}\right),
$$

is known for all $i$ and $j$ by Eq.(26), we apply the algorithm in Subsection 3.2 to recover the bit $u_{1}^{1}$ separately with two sets of $2^{34}$ frames sharing only one common frame denoted as the first frame for both sets. If we get a unique solution, we conclude the keystreams are generated by E0; otherwise, we accept them as truly random sources. The time/data complexities are $O\left(2 \times 2^{34} \times 5\right)$, i.e. $O\left(2^{37}\right)$. In contrast to the conventional treatment based on finding a multiple polynomial with low weight, no precomputation is needed in our scenario. So far, this is the only known near-practical attack against the full two-level E0. We can further improve the distinguisher by recovering $u_{t}^{1}$ for $t=1, \ldots, 4$ with two different sets of frames of the first 8 bits. Comparing two sets of solutions for the four bits, if we get a majority of identical bits, then we conclude the keystreams are generated by E0, otherwise we accept them as truly random sources. The number of frames we need is $2 \times 2^{34} / 4=2^{33}$. This results in time/data complexities $O\left(2^{33} \times 8\right)$, i.e. $O\left(2^{36}\right)$.

\subsection{The Key-Recovery Attack Against Two-Level E0}

From last subsection and Theorem[1, we know that with $2^{34}$ frames of keystreams, we can recover twelve bits, i.e. $u_{t}^{1}$ for $t=8 k+1, \ldots, 8 k+4, k=0,1,2$. After that, we try exhaustively for the remaining $|\mathcal{K}|-12$ bits assuming linear independency of the twelve bits 8 . Note that we have

$$
U^{i}=\left(G_{3} \circ M^{72} \circ G_{1}\right)\left(\mathbf{K}_{\mathbf{c}}^{\prime}\right) \oplus\left(G_{3} \circ M^{72} \circ G_{2}\right)\left(P^{i}\right),
$$

\footnotetext{
${ }^{8}$ We tested and found that the twelve bits are linearly independent for all choices of effective keylength $|\mathcal{K}|=8 \ell$ except for $|\mathcal{K}|=8$ in which case our attack is worse than the brute force attack and becomes meaningless anyway.
} 
by Eq.(612). So, we deduce from Eq.(1011) that

$$
U^{i}=\left(G_{3} \circ M^{72} \circ G_{1} \circ E\right)(\mathcal{K}) \oplus\left(G_{3} \circ M^{72} \circ G_{2}\right)\left(P^{i}\right),
$$

which means $U^{i}$ is an affine transformation of $\mathcal{K}$ given $P^{i}$ and so is $u^{i}$. Thus, we can ultimately solve the effective key $\mathcal{K}$ from $u^{i}$. The total time complexity of our attack is computed as

$$
2^{34}+2^{|\mathcal{K}|-13}= \begin{cases}2^{34}, & |\mathcal{K}|<48 \\ 2^{|\mathcal{K}|-13}, & |\mathcal{K}| \geq 48\end{cases}
$$

The data complexity of our attack is $\left(2^{34}-1\right) \cdot 24+128$, i.e. $O\left(2^{38.6}\right)$, as we need $2^{34}-1$ frames of the first 24 bits plus one frame of 128 bits. Table 3 compares our attack with existing attacks [15, 11, 1, 8, 9] against the two-level Bluetooth E0. Note that the number of required frames completely depends on the frame size in 7 to meet the requirement of data amount. This is, to our best knowledge, the first non-trivia 9 attack against practical E0 with various key length. Notice that when $40 \leq|\mathcal{K}| \leq 80$, our attack offers the best performance over the others.

Table 3. Comparison of our attack with existing attacks against two-level Bluetooth E0

\begin{tabular}{||c||c|c|c|c|c||}
\hline Attack & Precomputation & Time & Frames & Data & Memory \\
\hline \hline exhaustive search & - & $2^{|\mathcal{K}|-1}$ & 1 & $|\mathcal{K}|$ & - \\
\hline$[15$ & - & $2^{93}$ & 1 & $2^{7}$ & - \\
\hline$[1]$ & - & $2^{113}$ & 1 & $2^{7}$ & - \\
\hline$[7]$ & - & $2^{73}$ & - & $2^{43}$ & $2^{51}$ \\
\hline$[8]$ & $2^{80}$ & $2^{65}$ & 2 & $2^{12.4}$ & $2^{80}$ \\
\hline$[9]$ & $2^{80}$ & $2^{70}$ & 45 & $2^{17}$ & $2^{80}$ \\
\hline Our Attack & - & $2^{|\mathcal{K}|-13}+2^{34}$ & $2^{34}$ & $2^{38.6}$ & $2^{34}$ \\
\hline
\end{tabular}

Remark 2. Note that our attack is based on one of the largest two (linearly dependent) biases which is introduced in Eq.(3). As time/data tradeoff, we might also expect to have some other linearly independent biases to be large enough so that the time is decreased at somewhat reasonably increasing cost of data/memory complexities. Nonetheless, using the computation formula of [12, we find none such bias that leads to the data complexity of less than $2^{50}$.

Remark 3. As the nonces $P^{i}$ 's are affine transformation of a 26-bit clock and a master device address, our attack requiring much more than $2^{26}$ frames of keystreams still remains impractical unfortunately.

$\overline{9}$ in contrast to the brute force attack. 


\section{$5 \quad$ Extended Key-Recovery Attack Against Two-Level E0}

\subsection{A Partial Key-Recovery Attack}

Notice that on one hand, each of the four leftmost biased bits on the right-hand side of Eq. (9) is computed only with a certain subset of fixed key bits, the known nonce and the unknown variable FSM initial state; on the other hand, the value of Eq. (9) can be easily predetermined from the left-hand side, after we recover $u_{t}^{i}$, $s$ with $2^{34}$ frames by the distinguisher in Subsection 4.2. Consequently, the wellknown technique of statistical cryptanalysis leads us to the following approach to advance our key-recovery attack: supposing we manage to guess one of those four biased bits for all frames by guessing only the related key bits, then, for each frame, we XOR the guess on the biased bit with the predetermined value of Eq.(9) to obtain one bit. Thanks to Eq.(9), this bit shows bias for the right guess and almost balancedness for the wrong guess (which is also called statistical distinguishable); we're able to spot out the right guess of all guesses finally.

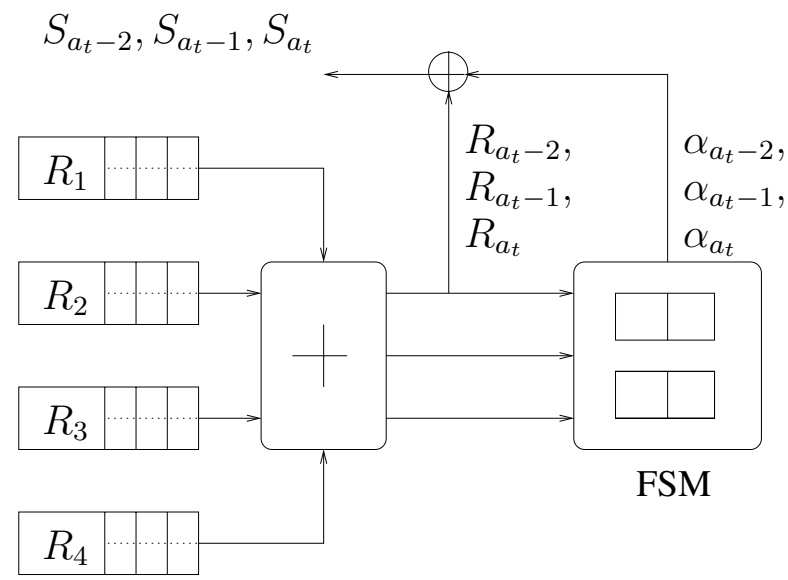

Fig. 2. Computation diagram of $S_{a_{t}-2}, S_{a_{t}-1}, S_{a_{t}}$

More specifically, we observe two important points about E0 FSM state: first, the FSM state at time $t$ always contains the two bits $c_{t}^{0}, c_{t-1}^{0}$; second, the 4-bit FSM state is updated by its current state together with four current output bits of LFSRs. Therefore, for fixed $t \in\{8 k+1, \ldots, 8 k+4\}$ and $k \in\{0,1,2\}$, the bit $10 \bigoplus_{j=0}^{4} \alpha_{a_{t}-j}^{i}$ computed from 5 consecutive bits $\alpha_{a_{t}}^{i}, \ldots, \alpha_{a_{t}-4}^{i}$, is derived from the same subset of $4 \times 3=12$ bits of the shared key $\mathcal{K}$ in all frames given $P^{i}$ together with the unknown frame-dependent FSM state at time $a_{t}-3$ (see

${ }^{10}$ For our convenience, we discuss the first biased bit on the right-hand side of Eq. (9) from now on; however, due to symmetry of the subscripts on the right-hand side of Eq. (9), our discussion also applies to the other three biased bits but the last. 
Fig. 21). We can compute all the possible sequences $\alpha_{a_{t}}^{\prime}, \ldots, \alpha_{a_{t}-2}^{\prime}$ in according to every possible FSM state for each frame $i$ with $t \in\{8 k+1, \ldots, 8 k+4\}$ and $k \in\{0,1,2\}$. Within one frame, of all the choices of 12 -bit $K^{\prime}$ of $\mathcal{K}$ and the FSM state, the sequence computed with the right shared 12-bit $K$ and the right FSM state yields $\bigoplus_{j=0}^{4} \alpha_{a_{t}-j}^{i}$, which equals $\bigoplus_{j=0}^{4}\left(\alpha_{a_{t}-j+8}^{i} \oplus \alpha_{b_{t}-j}^{i} \oplus \alpha_{b_{t}-j+8}^{i} \oplus \beta_{t+j}^{i}\right)$ with bias $\lambda^{4}$ when xoring with the computable bit $s_{t}^{i} \oplus u_{t}^{i}$ by Eq.(9); meanwhile, the sequence obtained with the wrong FSM state and/or the wrong shared 12-bit $K^{\prime}$ is expected to produce a new biased bit $\bigoplus_{j=0}^{4} \alpha_{a_{t}-j}^{\prime}$ (with bias $\lambda$ ) which when xoring with $s_{t}^{i} \oplus u_{t}^{i}$ finally generates a bit with much smaller bias $\lambda^{6}$ that could be approximated by a randomly and uniformly distributed bit. Therefore, we estimate that for every frame, the 12 -bit guess $K^{\prime}$ would yield $2^{4}$ randomly and uniformly distributed bits, except for the correct guess that produces $2^{4}-1=15$ randomly and uniformly distributed bits as well as one biased bit (with bias $\lambda^{4}$ ).

Alternatively, for every frame $i$, we can guess $4(2+\tau)$ bits $K^{\prime}$ of $\mathcal{K}$ together with the FSM state at time $a_{t}-\tau-2$ to compute consecutively $\tau$ bits $\bigoplus_{j=0}^{4} \alpha_{a_{t}-j}^{\prime}, \ldots, \bigoplus_{j=0}^{4} \alpha_{a_{t}-j-\tau+1}^{\prime}$ with $\tau \leq 5-(t \bmod 8)$ for fixed $t \in$ $\{8 k+1, \ldots, 8 k+4\}$ and $k \in\{0,1,2\}$. Denote the parameter $m$ as the required number of frames to be discussed later. For the same reason as before, when we xor the $\tau$ bits with $s_{t}^{i} \oplus u_{t}^{i}, \ldots, s_{t+\tau-1}^{i} \oplus u_{t+\tau-1}^{i}$, we expect the $16 m$ sequences to comply with a truly random distribution $\mathcal{D}_{0}$ of $\tau$-bit vectors for all wrong guesses $K^{\prime}$, and the $16 m$ sequences for the right guess $K$ to comply with the biased distribution $\mathcal{D}_{1}$ of $\tau$-bit vectors approximated by

$$
\mathcal{D}_{1} \approx \frac{\mathcal{D}^{\prime}+15 \mathcal{D}_{0}}{16}
$$

where $\mathcal{D}^{\prime} \stackrel{\text { def }}{=} \mathcal{D}^{\otimes 4}$ with $\otimes$ representing the regular convolutional product (see [12]), and $\mathcal{D}$ is the distribution of $\bigoplus_{j=0}^{4} c_{t-j}^{0}, \ldots, \bigoplus_{j=0}^{4} c_{t-\tau-j+1}^{0}$. Note that Eq.(11) means all the biases in $\mathcal{D}^{\prime}$ dwindles 16 times in $\mathcal{D}_{1}$, i.e. we have the following relation between the two Walsh coefficients $\hat{\mathcal{D}}_{1}(x), \hat{\mathcal{D}}^{\prime}(x)$ of any nonzero $\tau$-bit vector $x$ :

$$
\hat{\mathcal{D}}_{1}(x)=\frac{1}{16} \hat{\mathcal{D}}^{\prime}(x) .
$$

Let $f: G F(2)^{\tau} \rightarrow \mathbf{R}$ be a weighted grade for those resultant sequences $\chi_{K^{\prime}}^{1}, \ldots, \chi_{K^{\prime}}^{16 m}$ from the guess $K^{\prime}$. We accordingly grade each guess $K^{\prime}$ by

$$
G_{K^{\prime}}=\sum_{j=1}^{16 m} f\left(\chi_{K^{\prime}}^{j}\right) .
$$

Using analysis of [16] and 2] (see Appendix for complete treatment), we show that with minimal

$$
m \approx \frac{\tau+2}{2 \tau-1} \cdot 2^{34.5}
$$

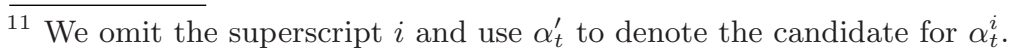


the score $G_{K}$ of the right guess tops the chart by choosing $f(x)=\mathcal{D}_{1}(x)-\frac{1}{16}$ for all $x \in G F(2)^{\tau}$. Note that with $f(x)=\mathcal{D}^{\prime}(x)$ we obtain equivalently the same resultant score $G_{K^{\prime}}$ for all $K^{\prime}$. Also, recall that to predetermine $u_{t}^{i}, \ldots, u_{t+\tau-1}^{i}$ we need $2^{34}$ frames for the distinguisher. Thus we must have

$$
m \approx \max \left(2^{34}, \frac{\tau+2}{2 \tau-1} \cdot 2^{34.5}\right) .
$$

Algorithm 1 details the above partial key-recovery attack for $4(2+\tau)$ bits.

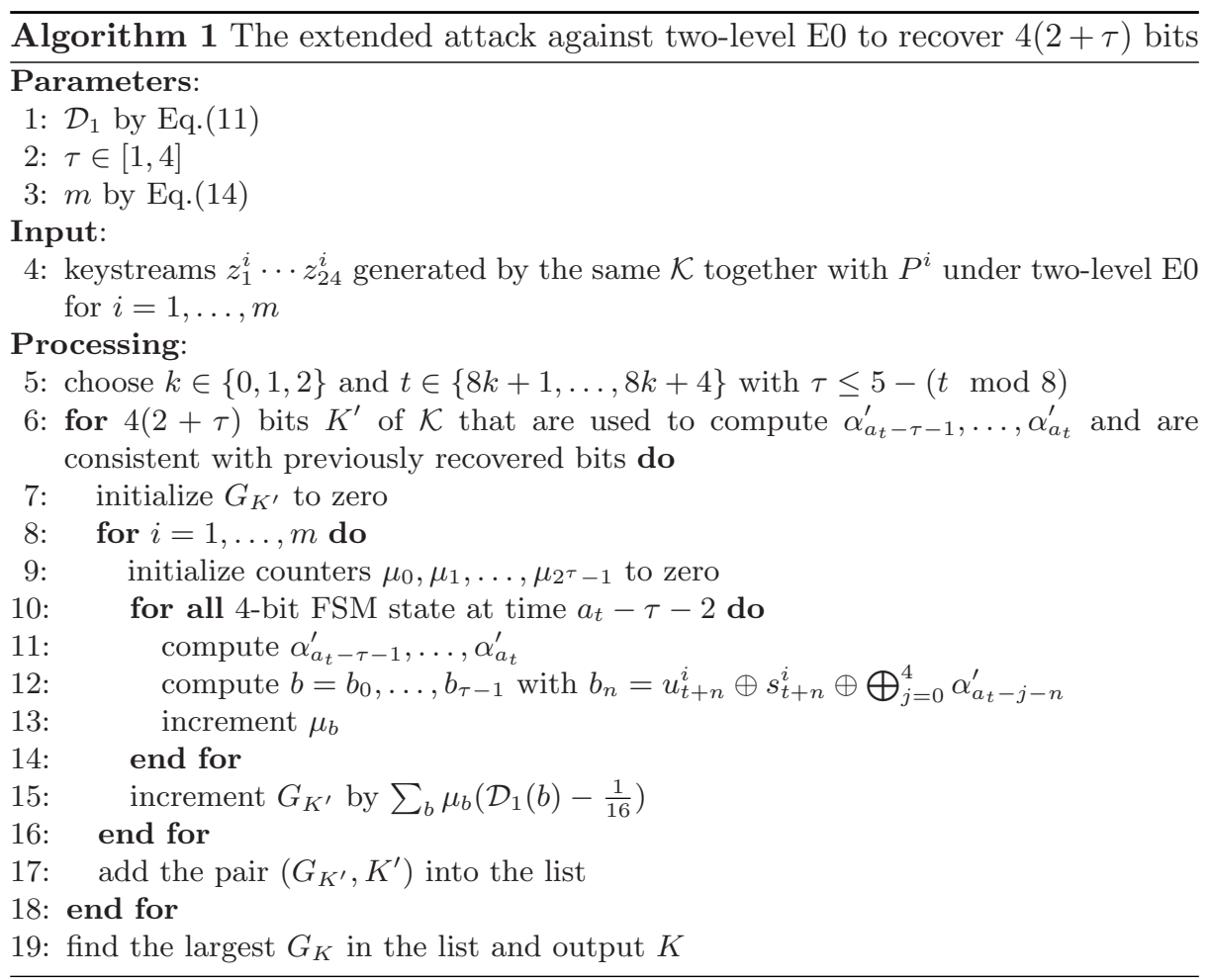

\subsection{Complexities and Optimization Issues}

About the performance of the partial key-recovery attack, it is seen from Algorithm 1 that to recover $4(2+\tau)$ bits, it runs $2^{4(2+\tau)} \cdot m \cdot 2^{4}(2+\tau)=m(2+\tau) \cdot 2^{12+4 \tau}$ times to compute each $\alpha_{t}^{\prime}$ for grading $2^{4(2+\tau)}$ candidates. In total, we have to perform $T=m(2+\tau) \cdot 2^{12+4 \tau}$ operations, where $m$ is set by Eq.(14).

Additionally, the loop from Line 9 to Line 15 can be done by one operation after precomputation as detailed below, which makes $T=m \cdot 2^{4(2+\tau)}$. During preprocessing, we run through every $y_{a_{t}-1}, \ldots, y_{a_{t}-\tau-2}$ (where $y_{t}$ denotes the Hamming weight of the original four component LFSRs' output bits at time $t$ ) to compute the $2^{4}$ possible sequences $\alpha_{a_{t}}^{\prime}, \ldots, \alpha_{a_{t}-\tau-1}^{\prime}$ which yield 
$2^{4}$ sequences $b^{\prime}=\bigoplus_{j=0}^{4} \alpha_{a_{t}-j}^{\prime}, \ldots, \bigoplus_{j=0}^{4} \alpha_{a_{t}-j-\tau+1}^{\prime}$ accordingly; and then for each $\tau$-bit $b^{\prime \prime}$, we increment the counter $\mu_{b^{\prime} \oplus b^{\prime \prime}}^{b^{\prime \prime}}$; last, we build up a table to store $h\left(y_{a_{t}-1}, \ldots, y_{a_{t}-\tau-2} ; b^{\prime \prime}\right)=\sum_{b^{\prime}} \mu_{b^{\prime} \oplus b^{\prime \prime}}^{b^{\prime \prime}}\left(\mathcal{D}_{1}\left(b^{\prime} \oplus b^{\prime \prime}\right)-\frac{1}{16}\right)$. The precomputation needs memory $2^{\tau} \cdot 5^{2+\tau} \approx 2^{3.32 \tau+4.6}$ and time $2^{4} \cdot 5^{2+\tau} \cdot(2+\tau) \cdot 2^{\tau} \approx$ $(\tau+2) 2^{3.32 \tau+8.6}$. After that, in real-time processing, for each frame $i$, we just compute $b^{i^{\prime \prime}}=b_{0}^{i^{\prime \prime}}, \ldots, b_{\tau-1}^{i^{\prime \prime}}$ with $b_{n}^{i^{\prime \prime}}=u_{t+n}^{i} \oplus s_{t+n}^{i}$ for $n=0, \ldots, \tau-1$, deduce $y_{a_{t}-1}^{i}, \ldots, y_{a_{t}-\tau-2}^{i}$ from $K^{\prime}, P^{i}$ and increment $G_{K^{\prime}}$ by $h\left(y_{a_{t}-1}^{i}, \ldots, y_{a_{t}-\tau-2}^{i} ; b^{i^{\prime \prime}}\right)$. Thus, we get $T=m \cdot 2^{4(2+\tau)}$.

Moreover, when $2^{4(2+\tau)} \cdot 2^{\tau} \leq m$, i.e. $2^{8+5 \tau} \leq m$, we can further reduce $T$ down to $m+2^{16+9 \tau}$. Notice that it is the same subset of $4(2+\tau)$-bit $\Omega^{i}$ of $P^{i}$ that is used to compute $y^{i}=\left(y_{a_{t}-1}^{i}, \ldots, y_{a_{t}-\tau-2}^{i}\right)$ with $K^{\prime}$. For convenience, let $g: G F(2)^{L} \rightarrow G F(2)^{4(2+\tau)}$ map $P^{i}$ to $\Omega^{i}$. We precompute a table $h^{\prime}(\Omega, q)$ for every $4(2+\tau)$-bit $\Omega$ and $\tau$-bit $q$ defined by:

$$
h^{\prime}(\Omega, q)=\sum_{i=1}^{m} \mathbf{1}_{\Omega=g\left(P^{i}\right), q=u_{t}^{i} \oplus s_{t}^{i}}
$$

with $u^{i}=\left(u_{t}^{i}, \ldots, u_{t+\tau-1}^{i}\right)$ and $s^{i}=\left(s_{t}^{i}, \ldots, s_{t+\tau-1}^{i}\right)$. This takes time $O(m)$ with memory $O\left(2^{8+5 \tau}\right)$. Recall that $u^{i}$ is determined independent of $K^{\prime}$ by the distinguisher in Subsection 4.2, and $u^{i}, s^{i}, y^{i}$ completely determine how to increment $G_{K^{\prime}}$ for frame $i$, i.e. by $h\left(y^{i} ; u^{i} \oplus s^{i}\right)$ from last paragraph. So, in real-time processing, for every $K^{\prime}$, instead of processing frame by frame to update $G_{K^{\prime}}$, we simply go through every $(8+5 \tau)$-bit pair $(\Omega, q)$, deduce $y=\left(y_{a_{t}-1}, \ldots, y_{a_{t}-\tau-2}\right)$ from $\Omega$ and $K^{\prime}$, then increment $G_{K^{\prime}}$ by $h^{\prime}(\Omega, q) h(y ; q)$. We reach the time complexity $T=m+2^{4(2+\tau)} \cdot 2^{8+5 \tau}=m+2^{16+9 \tau}$ for $2^{8+5 \tau} \leq m$.

To summarize, we have $T=m+2^{4(2+\tau)} \cdot \min \left(m, 2^{8+5 \tau}\right)$. Table 4 lists the best complexities of our partial key-recovery attack corresponding to $\tau=1, \ldots, 4$. Note that the success probability of the attack in the table is estimated according to the hypothesis test result of Eq.(11), i.e. the percentage of the $4(2+\tau)$-bit keys to generate a non-uniformly distributed sequence $\alpha_{a_{t}-\tau-3}^{\prime}, \ldots, \alpha_{a_{t}}^{\prime}$ with all the possible FSM state.

\subsection{The Overall Key-Recovery Attack}

Now we discuss how we proceed with the optimized Algorithm 1 to recover the full $\mathcal{K}$. With $\tau=2$ and fixed $k$, we independently run Algorithm 1 three times with $t=8 k+1, \ldots, 8 k+3$. And we expect at least two successes out of three

Table 4. Performance of our partial key-recovery attack against two-level E0

\begin{tabular}{||c||c|c|c|c||}
\hline$\tau$ & $\begin{array}{c}\text { Frames } \\
m\end{array}$ & $\begin{array}{c}\text { Time } \\
T\end{array}$ & $\begin{array}{c}\text { Prob. of } \\
\text { Success }\end{array}$ & $\begin{array}{c}\text { recovered key bits } \\
4(2+\tau)\end{array}$ \\
\hline \hline 1 & $2^{36}$ & $2^{36}$ & $50.8 \%$ & 12 \\
\hline 2 & $2^{35}$ & $2^{35}$ & $87.0 \%$ & 16 \\
\hline 3 & $2^{34.5}$ & $2^{43}$ & $99.0 \%$ & 20 \\
\hline 4 & $2^{34.3}$ & $2^{52}$ & $99.9 \%$ & 24 \\
\hline
\end{tabular}


runs. After checking consistency of all the overlapping bits for every possible pair of the algorithm outputs, we identify all the successful runs and obtain the minimum $16+4=20$ key bits.

We can easily adjust Algorithm 1 to target at any of the middle three biased bits on the right-hand side of Eq. (9) to recover 16 bits. With each modified partial key-recovery algorithm, we repeat previous procedure to recover minimum 20 bits. In total, we are sure to gather $4 \times 20=80$ bits. Since we already have 12 bits by the distinguisher, we finally exhaustively search the remaining $L-80-12=36$ bits within one frame. Algorithm 2 gives the abstract strategy of our complete attack. Therefore, to recover $L$-bit $\mathcal{K}$, our key-recovery attack works on $m=2^{35}$ frames, in time $24 m+4 \times 3 \times 2^{35} \approx 2^{40}$. The comparison of our attack with the best known attacks [7, 8, 9] against two-level E0 for $|\mathcal{K}|=L$ is available in Table 5.

Table 5. Comparison of our attack with the best attacks [7-9] against two-level E0 for $|\mathcal{K}|=L$

\begin{tabular}{||c||c|c|c|c|c||}
\hline Attack & Precomputation & Time & Frames & Data & Memory \\
\hline \hline$[7]$ & - & $2^{73}$ & - & $2^{43}$ & $2^{51}$ \\
\hline$[8]$ & $2^{80}$ & $2^{65}$ & 2 & $2^{12.4}$ & $2^{80}$ \\
\hline$[9]$ & $2^{80}$ & $2^{70}$ & 45 & $2^{17}$ & $2^{80}$ \\
\hline Our Attack & - & $2^{40}$ & $2^{35}$ & $2^{39.6}$ & $2^{35}$ \\
\hline
\end{tabular}

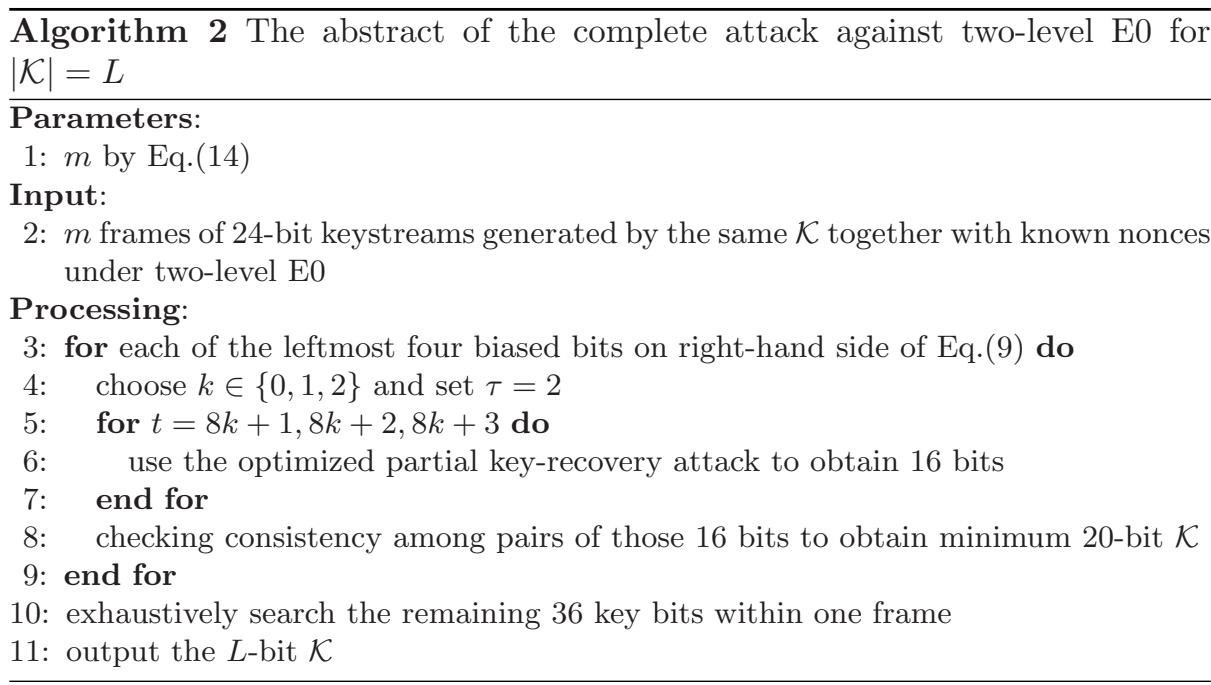

\section{Conclusion}

In this paper, based on one of the two largest biases inside the FSM within one-level E0, for the first time, we identify the bias at two-level E0 due to a 
resynchronization flaw in Bluetooth E0. Unlike the traditional approach to find the bias, the characterized bias does not involve the precomputation of the multiple polynomial with low weight. Second, to utilize the identified bias, we develop a novel attack to directly recover the original encryption key for two-level E0 without reconstructing the initial state of E0 at the second level. Our keyrecovery attack works with $2^{40}$ simple operations given the first 24 bits of $2^{35}$ frames. Compared with all existing attacks [15, 11, 7, 8, 9] against two-level Bluetooth E0, this is the best one so far, although the impossibly high amount of frames thwarts our attack to be practical. It remains an open challenge to decrease the data complexity with practical time and memory complexities. Finally, our attack illustrates the theory of statistical attacks in [2,16] with an example which is not based on linear cryptanalysis.

\section{Acknowledgments}

We owe a lot grateful thanks to Anne Canteaut and Willi Meier. And we would also like to thank the anonymous reviewers for many helpful suggestions.

\section{References}

1. Frederik Armknecht, Matthias Krause, Algebraic Attacks on Combiners with Memory, Advances on Cryptography - CRYPTO 2003, Lecture Notes in Computer Science, vol.2729, D. Boneh Ed., Springer-Verlag, pp. 162-175, 2003

2. Thomas Baignères, Pascal Junod, Serge Vaudenay, How Far Can We Go Beyond Linear Cryptanalysis?, in these proceedings

3. Bluetooth ${ }^{\mathrm{TM}}$, Bluetooth Specification, version 1.2, pp. 903-948, November, 2003, available at http://www.bluetooth.org

4. Philippe Chose, Antoine Joux, Michel Mitton, Fast Correlation Attacks: An Algorithmic Point of View, Advances in Cryptology - EUROCRYPT 2002, Lecture Notes in Computer Science, vol.2332, L. R. Knudsen Ed., Springer-Verlag, pp. 209-221, 2002

5. Nicolas T. Courtois, Fast Algebraic Attacks on Stream Ciphers with Linear Feedback, Advances on Cryptography - CRYPTO 2003, Lecture Notes in Computer Science, vol.2729, D. Boneh Ed., Springer-Verlag, pp. 176-194, 2003

6. Patrik Ekdahl, Thomas Johansson, Some Results on Correlations in the Bluetooth Stream Cipher, Proceedings of the 10th Joint Conference on Communications and Coding, Austria, 2000

7. Scott Fluhrer, Stefan Lucks, Analysis of the E0 Encryption System, Selected Areas in Cryptography- SAC 2001, Lecture Notes in Computer Science, vol. 2259, S. Vaudenay and A. Youssef Eds., Springer-Verlag, pp. 38-48, 2001

8. Scott Fluhrer, Improved Key Recovery of Level 1 of the Bluetooth Encryption System, available at http://eprint.iacr.org/2002/068

9. Jovan Dj. Golić, Vittorio Bagini, Guglielmo Morgari, Linear Cryptanalysis of Bluetooth Stream Cipher, Advances in Cryptology - EUROCRYPT 2002, Lecture Notes in Computer Science, vol. 2332, L. R. Knudsen Ed., Springer-Verlag, pp. 238-255, 2002 
10. Miia Hermelin, Kaisa Nyberg, Correlation Properties of the Bluetooth Combiner, Information Security and Cryptology- ICISC'99, Lecture Notes in Computer Science, vol. 1787, JooSeok. Song Ed., Springer-Verlag, pp. 17-29, 2000

11. Matthias Krause, BDD-Based Cryptanalysis of Keystream Generators, Advances in Cryptology - EUROCRYPT 2002, Lecture Notes in Computer Science, vol. 2332, L. R. Knudsen Ed., Springer-Verlag, pp. 222-237, 2002

12. Yi Lu, Serge Vaudenay, Faster Correlation Attack on Bluetooth Keystream Generator E0, Advances on Cryptography - CRYPTO 2004, Lecture Notes in Computer Science, vol.3152, M. Franklin Ed., Springer-Verlag, pp. 407-425, 2004

13. Mitsuru Matsui, Linear Cryptanalysis Method for DES Cipher, Advances in Cryptology - EUROCRYPT'93, Lecture Notes in Computer Science, vol.765, SpringerVerlag, pp. 386-397, 1993

14. Alfred J. Menezes, Paul C. van. Oorschot, Scott A. Vanstone, Handbook of Applied Cryptography, CRC, 1996

15. Markku Saarinen, Re: Bluetooth and E0, Posted at sci.crypt.research, 02/09/00

16. Serge Vaudenay, An Experiment on DES - Statistical Cryptanalysis, Proceedings of the 3rd ACM Conferences on Computer Security, pp. 139-147, 1996

\section{Appendix}

All our analysis here is similar with [16] and inspired by [2]. First, by Eq. (13), we have

$$
\operatorname{Exp}\left(G_{K^{\prime}}\right)=\frac{16 m}{2^{\tau}} \sum_{b} f(b)
$$

for a random wrong guess $K^{\prime}$, and by Eq.(11) we have

$$
\operatorname{Exp}\left(G_{K}\right)=\frac{15 m}{2^{\tau}} \sum_{b} f(b)+m \sum_{b} \mathcal{D}^{\prime}(b) f(b),
$$

for the right $K$. Hence,

$$
\Delta \operatorname{Exp}\left(G_{K^{\prime}}\right)=m \sum_{b}\left(\mathcal{D}^{\prime}(b)-\frac{1}{2^{\tau}}\right) f(b) .
$$

Meanwhile, we compute the variance of $G_{K^{\prime}}$ as

$$
\operatorname{Var}\left(G_{K^{\prime}}\right)=\frac{16 m}{2^{\tau}} \sum_{b}(f(b))^{2}-\frac{16 m}{2^{2 \tau}}\left(\sum_{b} f(b)\right)^{2}
$$

We can estimate the rank of $G_{K}$ over all possible $G_{K^{\prime}}$ by

$$
\operatorname{Exp}\left(\operatorname{Rank}_{G_{K}}\right) \approx 2^{4(2+\tau)} \Phi\left(-\frac{\Delta \operatorname{Exp}\left(G_{K^{\prime}}\right)}{\sqrt{2 \operatorname{Var}\left(G_{K^{\prime}}\right)}}\right) .
$$

In order to achieve the top rank for $G_{K}$, we see that the fraction 


$$
\frac{\Delta \operatorname{Exp}\left(G_{K^{\prime}}\right)}{\sqrt{\operatorname{Var}\left(G_{K^{\prime}}\right)}}
$$

must be large enough. This can be satisfied as long as the number $m$ of available frames is sufficiently large. However, aiming at a practical attack, we are concerned with the question of how to choose $f$ in order to minimize $m$ under the constraint of the top rank $G_{K}$. In order to maximize the fraction (18), we first maximize the numerator with the constraint that the denominator is a constant and then try to maximize the fraction over all the solutions. Define the multivariate polynomial

$$
g_{f}=m \sum_{b}\left(\mathcal{D}^{\prime}(b)-\frac{1}{2^{\tau}}\right) f(b)+\frac{16 m \gamma}{2^{\tau}} \sum_{b}(f(b))^{2}-\frac{16 m \gamma}{2^{2 \tau}}\left(\sum_{b} f(b)\right)^{2} .
$$

Using Lagrange's multiplier, we have

$$
\frac{\partial g_{f}}{\partial f(b)}=m\left(\mathcal{D}^{\prime}(b)-\frac{1}{2^{\tau}}\right)+\frac{32 m \gamma}{2^{\tau}} f(b)-\frac{32 m \gamma}{2^{2 \tau}} \sum_{b^{\prime}} f\left(b^{\prime}\right)=0,
$$

for all $b \in G F(2)^{\tau}$. From Eq.(19) we infer that

$$
\frac{f(b)-f\left(b^{\prime}\right)}{\mathcal{D}^{\prime}(b)-\mathcal{D}^{\prime}\left(b^{\prime}\right)}=\text { const. }
$$

for all $b \neq b^{\prime}$. Therefore we have a universal expression of $f$ as

$$
\frac{f(b)-\text { const. }}{\mathcal{D}^{\prime}(b)}=\text { const }^{\prime} .
$$

for all $b \in G F(2)^{\tau}$, which yields the same quantity of (18) regardless of the constants in $f$. So the easiest way to define $f$ could be $f(b)=\mathcal{D}^{\prime}(b)-\frac{1}{2^{\tau}}$ for all $b \in G F(2)^{\tau}$. Then Eq. (15) reduces to

$$
\begin{aligned}
\Delta \operatorname{Exp}\left(G_{K^{\prime}}\right) & =m \sum_{b}\left(\mathcal{D}^{\prime}(b)-\frac{1}{2^{\tau}}\right)^{2} \\
& =\frac{m}{2^{\tau}} \sum_{b \neq \mathbf{0}}(\hat{\mathcal{D}}(b))^{8} \\
& \approx \frac{m}{2^{\tau}}(2 \tau-1) \lambda^{8} .
\end{aligned}
$$

On the other hand Eq. (16) reduces to

$$
\operatorname{Var}\left(G_{K^{\prime}}\right) \approx \frac{16 m}{2^{2 \tau}}(2 \tau-1) \lambda^{8} .
$$


So we deduce from Eq.(17) that

$$
\begin{aligned}
\operatorname{Exp}\left(\operatorname{Rank}_{G_{K}}\right) & \approx 2^{4(2+\tau)} \Phi\left(-\frac{\lambda^{4}}{4} \sqrt{\frac{m(2 \tau-1)}{2}}\right) \\
& \approx \frac{2^{4(2+\tau)}}{\sqrt{2 \pi}} e^{-\frac{m(2 \tau-1)}{64} \lambda^{8}} .
\end{aligned}
$$

This means Rank $_{G_{K}}$ is expected to top the chart with

$$
m \approx \frac{256(2+\tau) \log 2}{\lambda^{8}(2 \tau-1)} \approx \frac{\tau+2}{2 \tau-1} \cdot 2^{34.5} .
$$

